\title{
Modelling of fast hydraulic transients: issues, challenges, perspectives
}

\author{
Yves ZECH ${ }^{1}$, Sandra SOARES-FRAZÃO², Sylvie VAN EMELEN ${ }^{3}$
}

\author{
1. Université catholique de Louvain, Ecole Polytechnique de Louvain, e-mail : yves.zech@uclouvain.be \\ 2. Université catholique de Louvain, Ecole Polytechnique de Louvain, e-mail : sandra.soares-frazao@uclouvain.be \\ 3. Fond de la Recherche Scientifique-FNRS, e-mail : sylvie.vanemelen@uclouvain.be
}

\begin{abstract}
The paper proposes an overview of different modelling approaches used for severe transients, particularly dam-break waves. One-, two- and three-dimensional approaches are briefly recalled with some examples in order to highlight their strengths and weaknesses, especially regarding the accurate speed of information through the numerical schemes. Two examples are developed with more details in order to highlight the result sensitivity to the selected model: the classical circular dam break and its dependency on the mesh, and the behaviour of the dam-break flow in a $90^{\circ}$ bend. Experimental data are also presented in order to show that, even in the near-field, hydrostatic pressure assumption has a limited impact on the accuracy. Some results from Smoothed Particle Hydrodynamics are also compared to common Eulerian approaches. In order to use the best of each approach, hybrid methods are also considered through the example of the $90^{\circ}$ bend.

Fast transients generally induce erosion and deposition of large amounts of sediment. The challenge is now to account for the inertia of the sediments suddenly mobilised. A two-layer model is proposed, with some assumptions, not wholly in line with experimental observations, but with promising results. Several improvements are tested but closure equations remain delicate and require more fundamental investigations.
\end{abstract}

Key-words: Dam-break, hybrid approaches, sheet flow, two-layer approach

\section{Modélisation des transitoires hydrauliques sévères : enjeux, défis, perspectives}

RÉSUMÉ. - Le papier propose un survol de différentes approches utilisées pour modéliser des transitoires sévères, en particulier l'onde de rupture de barrage ou de digue. Les principes des approches uni-, bi- ou tridimensionnelles sont rappelés avec leurs forces et leurs faiblesses, spécialement concernant la célérité de l'information au sein du schéma numérique. Deux exemples sont développés pour illustrer la sensibilité des résultats au choix du modèle : la rupture d'un barrage circulaire et l'onde de rupture dans un coude à $90^{\circ}$. Des mesures expérimentales sont aussi présentées pour montrer l'impact limité de l'hypothèse de distribution hydrostatique de pression. En vue de tirer bénéfice des avantages de chaque approche, des méthodes hybrides sont illustrées à travers l'exemple du coude à $90^{\circ}$.

Les transitoires sévères induisent généralement une grande quantité d'érosion et de dépôt. Dans ce cas, l'enjeu est de tenir compte de l'inertie des sentiments brusquement mobilisés. Un modèle à deux couches est proposé, avec des hypothèses non parfaitement conformes aux observations, mais avec des résultats prometteurs. De récentes pistes d'amélioration ont été testées mais les équations de fermeture demeurent délicates.

Mots-clés : Rupture de barrage, approches hybrides, modèles à deux couches

\section{INTRODUCTION}

The aim of the paper is to present an overview of modelling approaches used for dam- and dyke-breaking flows and to share some of the authors' experience in that field. This kind of problem is not only an academic game since important issues for decision makers rely on the accuracy of simulation tools in terms of alert and emergency organisation. In particular, two specific parameters have to be predicted as accurately as possible: the maximum water depth, and the arrival time of the wave front.

The accurate prediction of the arrival time is generally the most difficult to fulfil. This accuracy may significantly depend on the discretisation of the valley topography, but also on the interaction between the flow and its environment, particularly if the flooding occurs over loose bed and banks, which is generally the case of natural valleys.
The paper will be arranged in two sections. The first section deals with the selection of the most adequate model according to the characteristics of the valley. This includes some simplified downgraded models that can be used in order to make the modelling of heterogeneous valleys more affordable in terms of computational cost, for example in case of rugged geometry, sometimes difficult to discretise in details. The second section attempts to address the case of rapid transients over a mobile bed.

\section{HOW TO SELECT THE MOST ADEQUATE MODEL?}

The models discretise the geometry of the valley with various degrees of accuracy: the approach may be one-, two- or three-dimensional, using structured or unstructured 
meshes. The mathematical description may be Eulerian or Lagrangian. Also the numerical approaches may vary significantly from one case to another. The choice between these different approaches is not trivial and it is not always evident that the most complicated model is the most efficient. This section is not aimed at developing an exhaustive overview of all those approaches, but rather to present some examples of the issues and limitations related to a selection of the possible modelling approaches.

\section{II.1. One-dimensional (1D) approach}

This approach relies on the simplest representation of the valley geometry, described by a series of cross-sections at given space intervals along the valley thalweg (coordinate $x$ ). The key advantage of this type of model is that it does not require too many data. Moreover possible inaccuracies in the data do not influence too much the results.

However the mathematical description by the shallow-water (Saint-Venant) equations, based on the assumption of parallel velocities and hydrostatic pressure distribution, is not so simple in case of complicated geometries. These equations can be written in the following conservative form:

$$
\begin{gathered}
\frac{\partial A}{\partial t}+\frac{\partial Q}{\partial x}=0 \\
\frac{\partial Q}{\partial t}+\frac{\partial}{\partial x}\left(\beta \frac{Q^{2}}{A}+g I_{1}\right)=g A\left(S_{0}-S_{f}\right)+g I_{2}
\end{gathered}
$$

where $A$ is the cross-section area, $Q$ the discharge, $\beta$ the Boussinesq coefficient accounting for a non-uniform distribution of the velocity, $S_{0}$ is the bed slope and $S_{f}$ the friction slope, $I_{1}$ is the first moment of the cross-section accounting for the hydrostatic pressure on area $A$ (Fig. 1), and $I_{2}$ represents the effect of a spatial variation of the cross-section width, so accounting for the lateral pressure due to section enlargement or constriction:

$$
\begin{gathered}
I_{1}=\int_{0}^{h}(h-\eta) b(\eta) d \eta \\
I_{2}=A \frac{\partial h}{\partial x}-\frac{\partial I_{1}}{\partial x}
\end{gathered}
$$

So, the term $-g \partial I_{1} / \partial x$ represents the difference between the hydrostatic pressure forces on both ends of the control volume (Fig. 2), from which $g I_{2}$ is the part due to a change in the cross-section width, which is counterbalanced by the longitudinal component of the pressure exerted on the lateral surface of the reach.

The form (1-2) of the equations is conservative, so ready to use for the common finite-volume numerical scheme, written in the general vector form:

$$
\frac{\partial \mathbf{U}}{\partial t}+\frac{\partial \mathbf{F}(\mathbf{U})}{\partial x}=\mathbf{S}(\mathbf{U})
$$

where $\mathbf{U}$ is the vector of conserved variables, here $(A, Q)^{\mathrm{T}}$, and $\mathbf{F}$ is the flux term consisting of the fluxes of mass and momentum, this latter combined with the pressure variation due to water depth variation: $g \partial I_{1} / \partial x$, while the pressure variation due to non prismaticity, represented by $I_{2}$, is relegated in the source term $\mathbf{S}$, since not depending on the conserved variables.

In finite-volume formulation, (5) leads to the following discrete scheme around a cell $i$, located between cells $i-1$ and $i+1$ :

$$
\mathbf{U}_{i}^{n+1}=\mathbf{U}_{i}^{n}-\frac{\Delta t}{\Delta x}\left(\mathbf{F}_{i+1 / 2}^{*}-\mathbf{F}_{i-1 / 2}^{*}\right)+\mathbf{S}_{i}^{*} \Delta t
$$

where superscripts $n$ and $n+1$ refer to the initial and final time of the time step and $*$ to an intermediate time to be defined (Fig. 3). In the first-order scheme, the variables are considered as constant in the computational cell (Fig. 4), resulting in discontinuities at the cell interfaces. These discontinuities form Riemann problems from which the fluxes $\mathbf{F}^{*}$ between computational cells can be evaluated.

One of the common difficulties is to avoid spurious flow when computing water at rest over an irregular topography. Indeed, the flux term $\mathbf{F}$, including the pressure variation, is generally calculated using an upwind scheme that gives more weight to the upstream cell. The source term $\mathbf{S}$, including the part of the pressure due to non-prismaticity, needs then to be discretised according to the $C$-property [Bermudez and Vasquez, 1994], i.e. in a way that is compatible with the pressure terms in the fluxes $\mathbf{F}$ to avoid creation of spurious momentum. This property is of particular importance if the valley is non-prismatic, with $I_{2} \neq 0$ [Petaccia et al., 2013].

Another issue, directly related to fast transients and particularly to dam-break problems, is the speed of information. This is accurately represented by characteristics that precisely follow the propagation of information within the

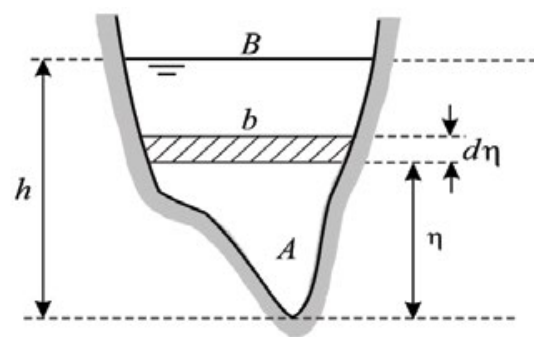

Figure 1 : Definition of $1^{\text {st }}$ moment.

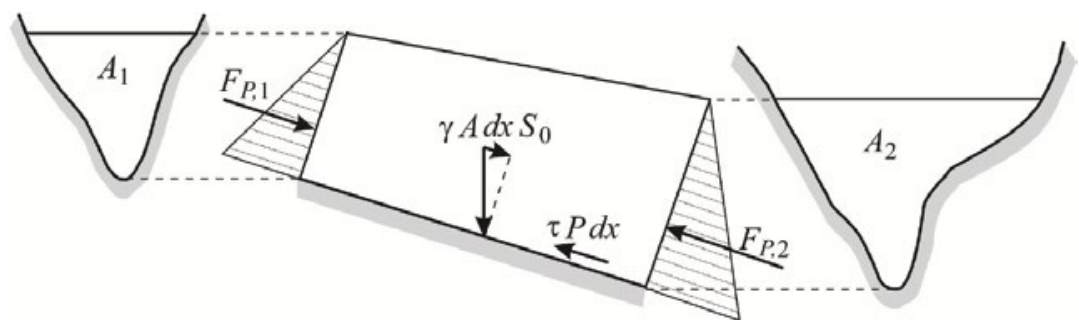

Figure 2 : Equilibrium of a control volume. 


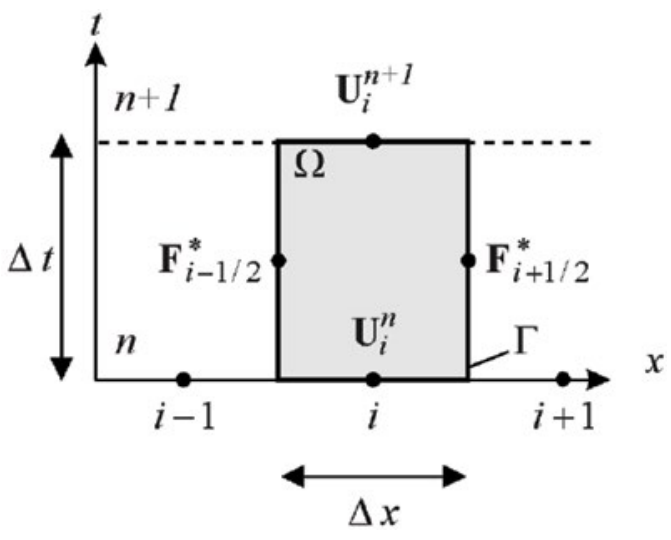

Figure 3 : Finite-volume scheme.

system. These characteristics are the basis of most of the flux predictors $\mathbf{F}^{*}$ required by finite-volume schemes such as (6). For instance, the Pavia scheme illustrated in Fig. 5 [Braschi and Gallati, 1992]

$\mathbf{F}_{i+1 / 2}^{*}=\frac{1+\overline{\mathrm{F}} \mathrm{r}}{2} \mathbf{F}_{i}^{n}+\frac{1-\overline{\mathrm{F}} \mathrm{r}}{2} \mathbf{F}_{i+1}^{n}+\bar{c} \frac{1-\overline{\mathrm{F}} \mathrm{r}^{2}}{2}\left(\mathbf{U}_{i}^{n}-\mathbf{U}_{i+1}^{n}\right)$

is directly issued from the characteristics. In (7), $\overline{\mathrm{F}} \mathrm{r}=\bar{V} / \bar{c}$ is the Froude number, the overbar indicating an averaging between left and right cell values, $c=(g A / B)^{1 / 2}$ being the wave celerity and $V=Q / A$ the mean velocity. To make the expression valid also for supercritical flow, the value of $|\bar{F} r|$ is limited to 1 , in such a way that the flux becomes fully upwinded when $|\overline{\mathrm{F}} r| \geq 1$. The last term in (7), active only when $|\overline{\mathrm{Fr}}|<1$ represents a kind of diffusion that vanishes for supercritical flow.

Actually, even if derived in various ways, the most popular flux predictors, for instance Roe scheme [Roe, 1981] and HLL solver [Harten et al, 1983; Guinot, 2008] can be written in the same form as (7).

As the flux predictor is based on characteristics, a correct propagation of information and waves is ensured. However, in a dam-break flow over dry bed, the transmission of the information is essentially guided by the cell arrangement.

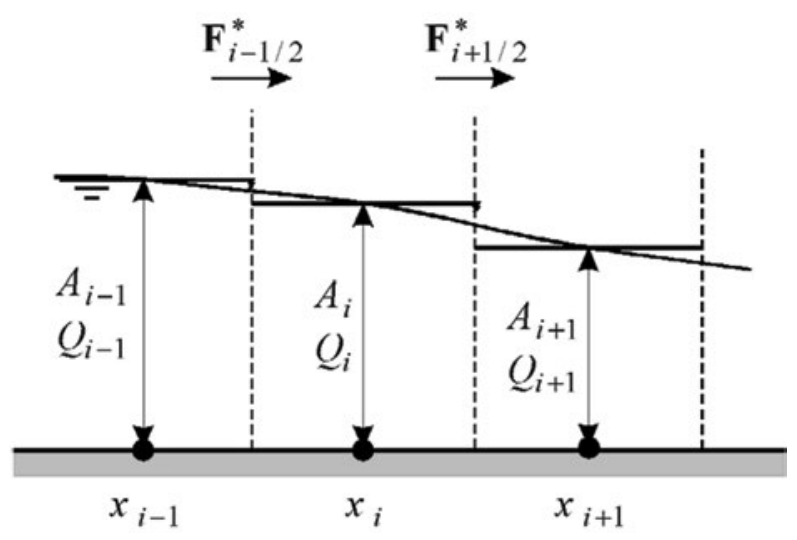

Figure 4 : Definition of integration cells.

This is illustrated in Fig. 6 [Soares-Frazão, 2002]: without specific precaution, at each time step, a new cell will receive upstream information, in such a way that the front speed will depend on the space interval $\Delta x$.

According to the Ritter analytical solution for a dam break with initial water depth $h_{0}$ in the upstream reservoir, at the wave front the celerity and the water velocity are equal to $2\left(g h_{0}\right)^{1 / 2}$, while the water depth and the discharge are equal to 0 . But as the variables are precisely the water depth $h$ and the unit discharge $q$, the computed velocity $q / h \rightarrow 0 / 0$. However, in practice, as $h \rightarrow 0$ faster than $q \rightarrow 0$, the computed velocity might tend toward infinity and the numerical simulation will fail. One solution [Soares-Frazão, 2002] could be to impose a minimum water depth $h_{m i n}$, and to block the flux until this depth is reached in the downstream cell (Fig. 6). With this numerical trick that ensures stability of the scheme for wave propagation over a dry be, the front speed still depends on the space interval $\Delta x$, but in a less pronounced way, and also on the value of $h_{\min }$. A comparison between the analytical and numerical front wave propagation speed is shown in Fig. 7 for different values of $h_{\text {min }}$ : it can be observed that the computed front celerity is always slower than the analytical one.

In conclusion, accurate following of the wave front, although crucial for alerting threatened population, remains a difficult task in one-dimensional approach. This task maybe still more complex for two- or three-dimensional approaches.
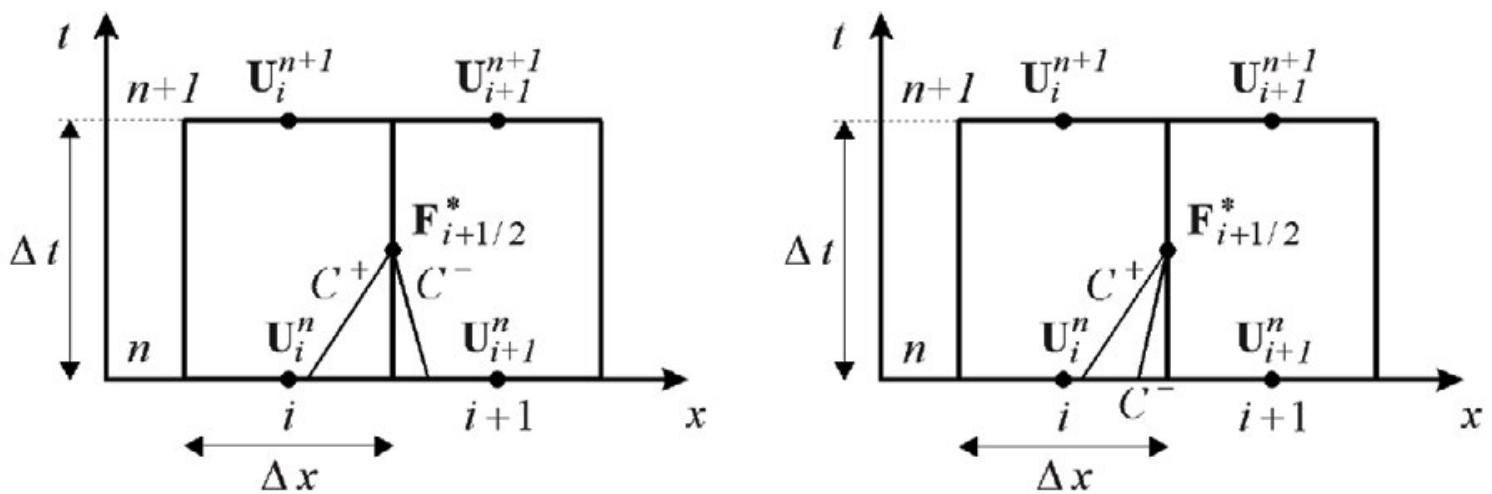

Figure 5 : Pavia scheme for flux computation (left: subcritical; right: supercritical flow). 


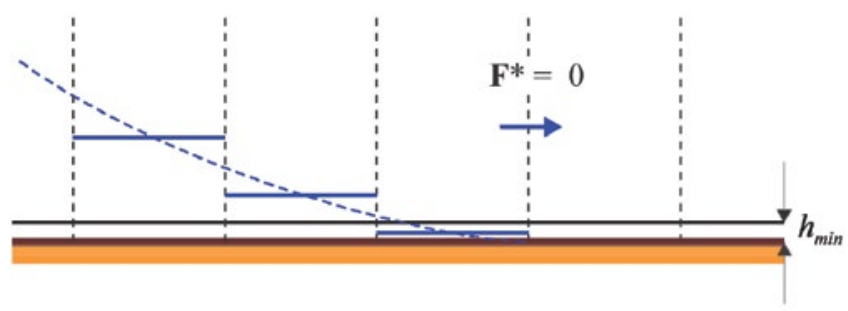

Figure 6 : Wave front on a dry bed (the dotted line is the analytical solution of an idealised dam break).

\section{II.2. Two-dimensional (2D) approach}

In two-dimensional conservative vector form the SaintVenant shallow-water equations read [e.g. Guinot, 2008]:

$$
\frac{\partial \mathbf{U}}{\partial t}+\frac{\partial \mathbf{F}(\mathbf{U})}{\partial x}+\frac{\partial \mathbf{G}(\mathbf{U})}{\partial y}=\mathbf{S}
$$

with $\mathbf{U}=\left(\begin{array}{c}h \\ u h \\ v h\end{array}\right), \mathbf{F}=\left(\begin{array}{c}u h \\ u^{2} h+g h^{2} / 2 \\ u v h\end{array}\right), \mathbf{G}=\left(\begin{array}{c}v h \\ u v h \\ v^{2} h+g h^{2} / 2\end{array}\right)$,

$\mathbf{S}=\left(\begin{array}{c}0 \\ g h\left(S_{0 x}-S_{f x}\right) \\ g h\left(S_{0 y}-S_{f y}\right)\end{array}\right)$

where $u$ and $v$ are the depth-averaged velocity components, and the subscript $x$ and $y$ denoting the component of the bottom slope $S_{0}$ and the friction slope $S_{f}$. A priori, this formulation is simpler than the $1 \mathrm{D}$ one, as it avoids the sometimes complex link between the cross-section area $A$ and the depth $h$. However, the price to pay for this simplicity is the considerable amount of data required for representing the valley geometry, and also the dependence of the results on the 2D grid selected for representing the problem.

Fig. 8 shows the example of a sinuous Belgian valley, for which dam-break flow computations were carried out

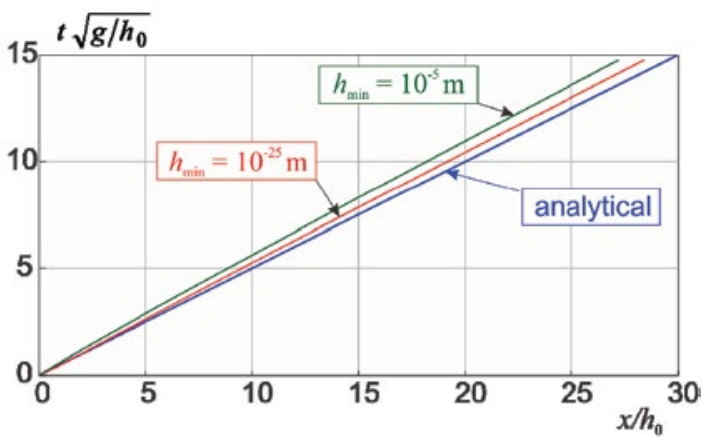

Figure 7 : Comparison between analytical and numerical front speed for different values of $h_{\text {min }}$

15 years ago with a limited data set, in such a way that the 2D approach was only possible with a rather coarse grid. The arrival time at the city of Malmedy was estimated equal to 6 minutes using a 1D model, but 45 minutes with a $40-\mathrm{m}$ square grid, this latter inducing significant but non-physical head losses. The use of a $60-\mathrm{m}$ triangular grid, more adapted to the valley shape but still coarse due to the poor data density, led to a 17-minute prediction, more realistic but not completely convincing. So, a 2D model requires a lot of data and an appropriate grid arrangement to be usable.

Moreover, the question of the information speed is somewhat delicate in 2D models. The domain of dependence, which was limited by two characteristics in 1D approach, is now a conical characteristic surface in the phase space $(x, y, t)$. Any point inside this surface may influence the point at the summit of the cone [Guinot, 2008]. The problem is generally circumvented by a secant plane approach. Equations (8), as most of the equation in fluid mechanics, are invariant by rotation and it is possible to use a projection of these equations onto a plane, for example normal to the interface $j$ of the triangular computation cell $i$ (Fig. 9), in order to restrict the 2D approach to a one-dimensional.

Defining $\mathbf{T}$ as the transformation matrix from the coordinate system $(x, y)$ to the local system $\left(x_{n}, y_{t}\right)$ The corresponding finite-volume scheme reads for a cell $i$ of base area $\Omega_{i}$ limited by $n b$ interfaces of lengths $L_{j}$ :

$$
\mathbf{U}_{i}^{n+1}=\mathbf{U}_{i}^{n}-\frac{\Delta t}{\Omega_{i}} \sum_{j=1}^{n b} \mathbf{T}_{j}^{-1} \mathbf{F}_{j}^{*}\left(\overline{\mathbf{U}}_{j}\right) L_{j}+\mathbf{S}_{i} \Delta t
$$
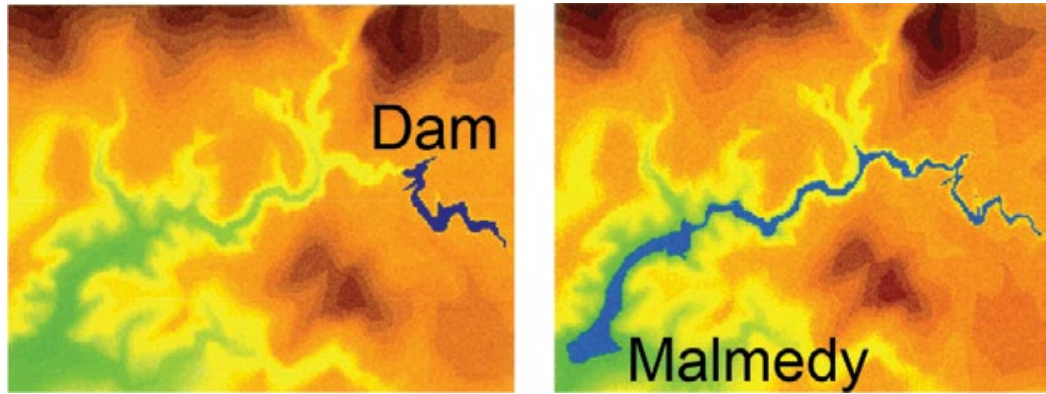

Figure 8 : Dam-break simulation in the Warche Valley (Belgium). 


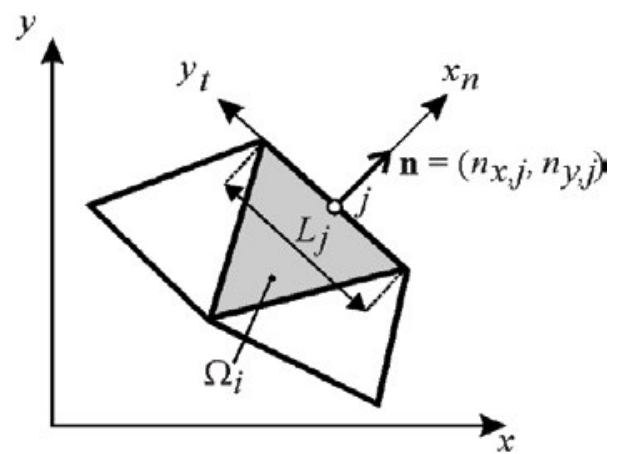

Figure $9:$ Riemann problem in $2 D$ approach.

$$
\begin{aligned}
& \text { with } \overline{\mathbf{U}}=\mathbf{T} \mathbf{U}=\left(\begin{array}{ccc}
1 & 0 & 0 \\
0 & n_{x} & n_{y} \\
0 & -n_{y} & n_{x}
\end{array}\right)\left(\begin{array}{c}
h \\
u h \\
v h
\end{array}\right)=\left(\begin{array}{c}
h \\
u_{n} h \\
v_{t} h
\end{array}\right) \text {, which implies } \\
& \mathbf{F}(\overline{\mathbf{U}})=\left(\begin{array}{c}
u_{n} h \\
u_{n}^{2} h+g h^{2} / 2 \\
u_{n} v_{t} h
\end{array}\right) \quad(9 b-c)
\end{aligned}
$$

The flux predictors now implies three characteristics, for which the propagation speeds are $u_{n}-(g h)^{1 / 2}, u_{n}$ and $u_{n}+(g h)^{1 / 2}$, respectively. Adapted flux F-predictor schemes can then be derived, such as for example the HLLC solver [Toro, 1997] that leads to relations similar to (7) for the two first components of vector $\mathbf{F}$ but a simple advection for the third component $\left(u_{n} v_{t} h\right)$.

However, in a discretised form, all the projection operations obviously alter the transmission of information, which is clearly visible in the classical example of circular dam break. Fig. 10 shows the computation of the wave due to a cylindrical water column of $2 \mathrm{~m}$ diameter with a water depth of $2 \mathrm{~m}$ collapsing in a 1-m deep water outside the reservoir. A finite-volume numerical scheme is used with a HLLC solver. An orthogonal structured mesh privileges the main directions, while an unstructured mesh results in a more uniform wave propagation, but without preventing a significant delay compared to a radial-circular mesh perfectly aligned with the wave propagation.

Of course, some techniques are developed to circumvent this type of limitation, generally relying on a more sophisticated description of the variables inside the computation cell. Among others, the EigenVector-based Reconstruction (EVR) approach, proposing a linear reconstruction of the water surface and taking advantage of the eigenstructure of the equations, seems particularly efficient [Soares-Frazão and Guinot, 2007].

However, it must be kept in mind that a $2 \mathrm{D}$ description is not a guarantee of accuracy, as it is partly depending on the skills of the mesh designer to anticipate the main flow direction.

\section{II.3. Three-dimensional (3D) approach}

Most of fast hydraulic transients present three-dimensional features, mainly in the near-field of the initiating phenomenon (sudden release of water, hydropower operations, damor dike-break), but also in the presence of obstacles (sudden change in the valley direction, bridge piers, etc.). So the ultimate dream of the modeller is of course to use a 3D model in order to capture all the detailed features of the flow, and also to predict the pressure exerted by the wave against the obstacles.

The 3D models rely on the mass and momentum conservation equations [Ferziger and Perić, 2002]:

$$
\begin{gathered}
\frac{\partial \bar{u}_{j}}{\partial x_{j}}=0 \\
\frac{\partial \bar{u}_{i}}{\partial t}+\frac{\partial \bar{u}_{j} \bar{u}_{i}}{\partial x_{j}}=-\frac{1}{\rho} \frac{\partial \bar{p}}{\partial x_{i}}+\frac{1}{\rho} \frac{\partial \bar{\tau}_{i j}}{\partial x_{j}}+g_{i}
\end{gathered}
$$

where $x_{i}(i=1,2,3)$ are the Cartesian coordinates, $u_{i}=\bar{u}_{i}+u_{i}{ }^{\prime}$ the velocity components decomposed in time-average and fluctuation velocity, $\bar{p}$ the time-averaged pressure, $\bar{\tau}_{i j}$ the time-averaged $i$-component of the stress (Reynolds stresses) on a face normal to $j$-direction, $g_{i}$ the $i$-component of the gravity. The internal stresses are mainly due to turbulence, of which the effect may be commonly represented as an increased viscosity. This leads to the eddy-viscosity model for the Reynolds stresses:

$$
\bar{\tau}_{i j}=-\rho \overline{u_{i}{ }^{\prime} u_{j}{ }^{\prime}}=\mu_{t}\left(\frac{\partial \bar{u}_{i}}{\partial x_{j}}+\frac{\partial \bar{u}_{j}}{\partial x_{i}}\right)-\frac{2}{3} \rho k \delta_{i j}
$$

where $k$ is the turbulent kinetic energy.

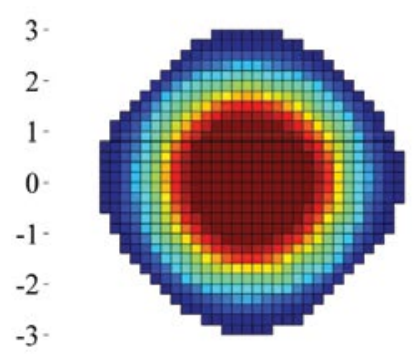

Rectangular $0.20 \mathrm{~m}$

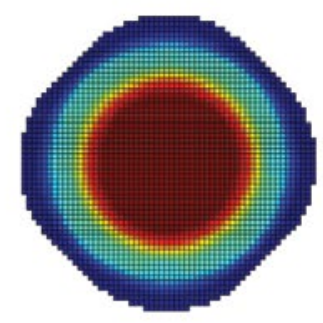

Rectangular $0.10 \mathrm{~m}$

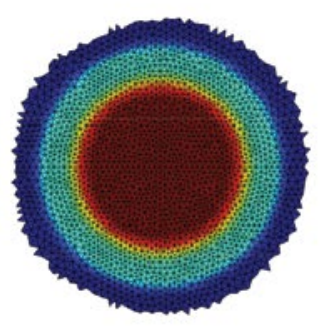

Unstructured mesh

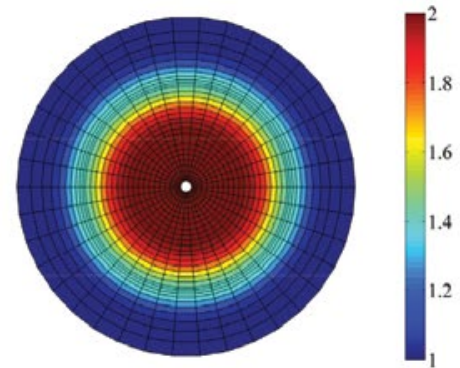

Radial mesh

Figure 10 : Circular dam break computed with different grids (left: distances in m; right: colour bar level in m). 


$$
k=\left(\overline{u_{1}^{\prime} u_{1}{ }^{\prime}}+\overline{u_{2}{ }^{\prime} u_{2}{ }^{\prime}}+\overline{u_{3}{ }^{\prime} u_{3}}\right) / 2
$$

and $\delta_{i j}$ the Kronecker symbol, $\mu_{t}$ being the eddy viscosity that can be estimated by various models, among which the popular $k$ - $\varepsilon$ model.

To capture the evolution of the water surface, it is common to freeze this one which is thus treated as a solid limit, then to compute the pressure distribution along this fictitious limit and to move the water surface geometry in order to recover a null pressure along this. This technique is of course not adapted to fast transients where the water surface may evolve significantly from one time step to the other. So, most of the models circumvent this difficulty by using a volume-of-fluid (VOF) technique. The VOF method is a surface-tracking technique, in which a specific transport equation is used to determine the relative volume fraction of water $\eta$ in each computational cell [Biscarini et al., 2010]. So, $\eta$ is in the range $[0,1]: 0$ in air, 1 in water, between 0 and 1 in a cell containing the water surface, depending on the surface position. A term is now added to the motion equation (10b) to account for the surface curvature and the resulting surface tension, and a transport equation is added to $(10)$ for the transport of the relative water fraction:

$$
\frac{\partial \eta}{\partial t}+\frac{\partial \bar{u}_{j} \eta}{\partial x_{j}}=0
$$

In the $3 \mathrm{D}$ approach, the internal stresses are explicitly described, while the 1D and 2D approaches rely on a simplified model of the bed- and bank-induced turbulence by means of the energy slope $S_{f}$ estimated for instance from a Manning-Strickler equation. So, 3D approaches are designed to account for and represent any recirculation zones in the flow, while 2D can only predict horizontal recirculation, as far as depth-averaged shear stresses are considered. Such circulation can appear for example in a sudden expansion of the valley. However, in the case of a dam break, the influence of a recirculation on the main stream is generally negligible [Soares-Frazão et al., 2003]. Moreover, numerical diffusion often induces artificial recirculation, although the results in such a zone are of course not accurate.

An obvious advantage of the $3 \mathrm{D}$ approach is the possibility to account for the vertical component of the velocity and for a non-hydrostatic pressure distribution that arises from the non-parallel flow and from stream line curvature. However, even in the near-field of an idealised dam break, the development of the non-parallel zone is rather limited. Recent experiments [Aleixo et al., 2011; Aleixo et al., 2013] have explored the velocity field in the vicinity of a dam-break simulated by a gate rapidly moving down. Fig. 11 shows some of their results for an experiment with initial water depth $h_{0}=0.325 \mathrm{~m}$ in the upstream reservoir. Two situations are depicted: at non-dimensional time $t /\left(h_{0} / g\right)^{1 / 2}=2.50$ and 5.00 , respectively. For this latter time, the distribution in angle and amplitude of the velocities is also shown, clearly indicating that the velocities, while not completely horizontal, are parallel enough to justify an assumption of hydrostatic pressure distribution and the use of shallow-water equations.

Regarding the speed of information, 3D modelling is often rough. In $(10 b)$, the second term of the left-hand side represents the convection, while the second derivatives of the right-hand side, obtained introducing (11) in (10b), represents the diffusion. According to Patankar [1980], the non-dimensional Peclet number representing the ratio of the strength of convection and diffusion is used for deciding the degree of upwinding. Beyond a given threshold of the Peclet number the upwinding is forced to be complete, while, below this threshold value the upwinding is only partial, according to various schemes. This idea is directly inspired by heat transfer between two bodies of distinct temperatures. That means that the Peclet number plays the role in $3 \mathrm{D}$ approach that the Froude number plays in $2 \mathrm{D}$. So, the speed of information is also depending on the size and arrangement of computational cells, with the risk of spurious delays, if the cells are not well aligned with the main information paths.

A typical example of three-dimensional behaviour of a fast-transient flow is the experiment of a dam-break flow in a channel with a $90^{\circ}$ bend carried out at the Université catholique de Louvain [Soares-Frazão and Zech, 2002]. Fig. 12 illustrates the experimental set-up, consisting of an upstream reservoir of $2.44 \mathrm{~m} \times 2.39 \mathrm{~m}$, a $0.495 \mathrm{~m}$ wide rectangular flume with glass walls, composed of a $\sim 4 \mathrm{~m}$ long upstream reach, a $90^{\circ}$ bend, and a following $\sim 3 \mathrm{~m}$ long downstream reach. The channel bed level is $0.33 \mathrm{~m}$ above the upstream reservoir bed level. The downstream end of the channel is open. In the bend, a sudden rise of the water occurs that can be explained from the energy point of view. In the initial supercritical stage, the kinetic energy
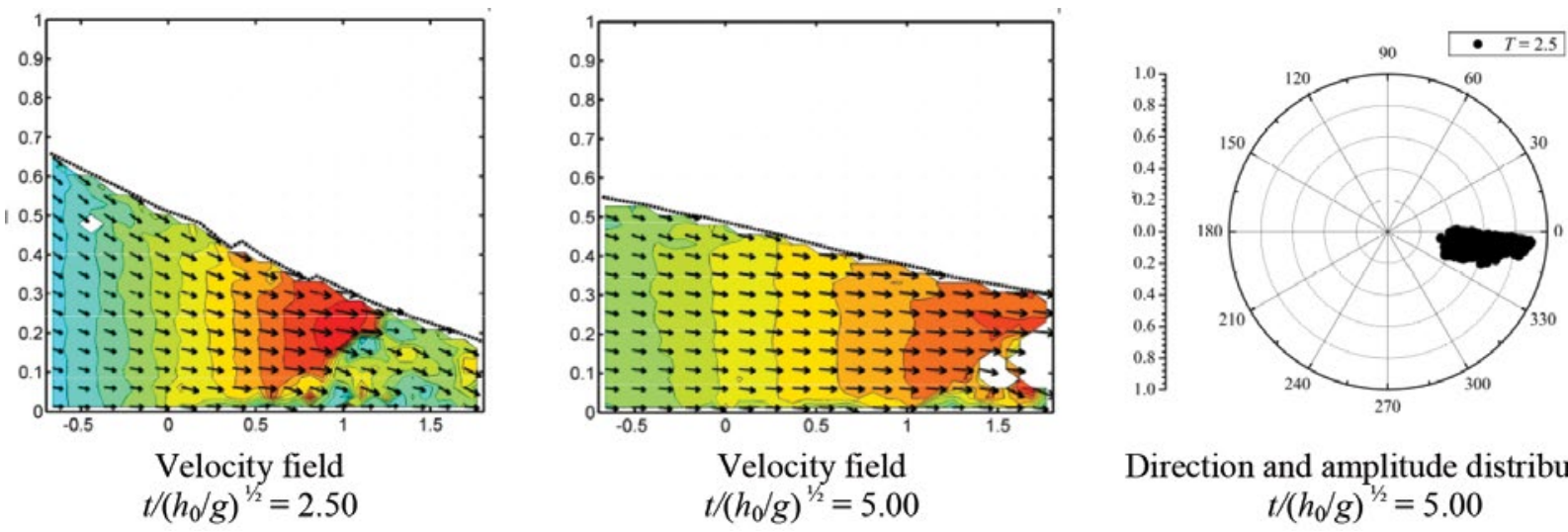
Direction and amplitude distribution
\[ t /\left(h_{0} / g\right)^{1 / 2}=5.00 \] $t /\left(h_{0} / g\right)^{1 / 2}=5.00$

Figure 11 : Velocity distribution in the dam-break near-field (non-dimensional graduation: $x / h_{0}, z / h_{0}$ ). The initial gate position is at $x=0$. 
is predominant. Then, when the water is suddenly stopped in the bend, its kinetic energy is transformed into potential energy (rise of the water surface) and important head losses occur. Contrarily to kinetic energy, which is associated to velocity vectors with a given direction, the potential energy is purely scalar without any preferential direction. This potential energy then transforms back into kinetic energy: a part of the water flows further downstream, while another part recedes upwards to the reservoir developing a bore. This is illustrated in Fig. 13.

Fig. 14 represents the water profile at two characteristic times, obtained by means of digital imaging technique (black lines). Water profiles were acquired at different view windows along the channel and then arranged to form a developed flow profile that can be compared with the computed ones. The bend zone, located between abscissa $6.31 \mathrm{~m}$ and $7.3 \mathrm{~m}$, is indicated by two vertical lines. Two computational results are shown: a 2D finite-volume approach by the authors [Soares-Frazão and Zech, 2002] using a Roe solver, and a 3D VOF approach by Biscarini et al. [2010]. The experimental data present some gaps, due either to blind zones by the channel structure, or to the splash effect of the wave arrival against the bend. The 3D model, while producing spurious surface waves, seems significantly better for the first water rebound, but with a trend to smoothen the receding bore occurring at $t=7 \mathrm{~s}$. The $2 \mathrm{D}$ model is better regarding this point but with some delay in the propagation speed of the receding bore. So, both approaches seem complementary.

Besides the classical VOF method, some less conventional approaches are also investigated. In particular, the Smoothed Particle Hydrodynamics (SPH) is a mesh-free method, where the fluid domain is represented by a set of irregularly spaced nodal points where the physical properties are known. These nodal points then move with the fluid hence making the technique a Lagrangian method. The physical properties of each particle can change with time due to the interactions with neighbouring particles. This is translated in the numerical scheme by means of weighting functions, sometimes delicate to define near the free surface [Gomez-Gesteira et al., 2010]. Fig. 15 shows the result of such a method for a body of water 2-m deep and 1-m long collapsing in a reservoir 3-m long. The SPH results by Gomez-Gesteira et al. [2010] are compared to a simple 1D approach, with two different assumptions regarding the bed friction.

The SPH results appear delayed, showing that the speed of information remains problematic, as an artificial viscosity has to be imposed to prevent instabilities, without clear link with physical friction coefficients. Another observation is that SPH

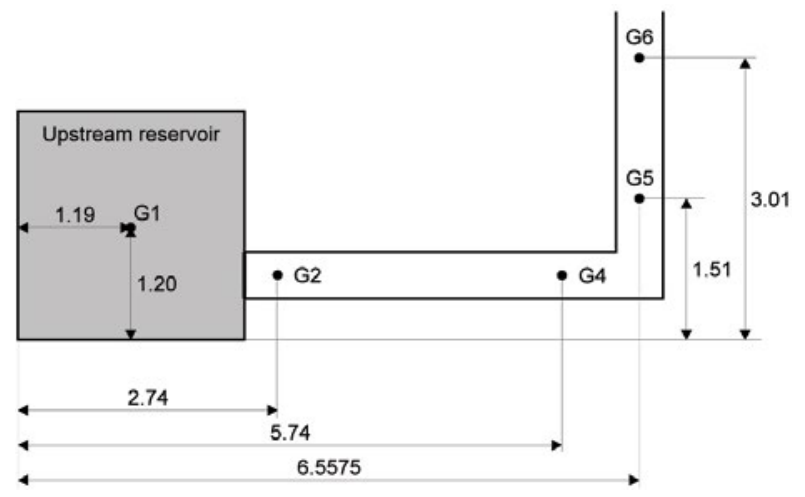

Figure 12: $90^{\circ}$ bend experimental set-up.
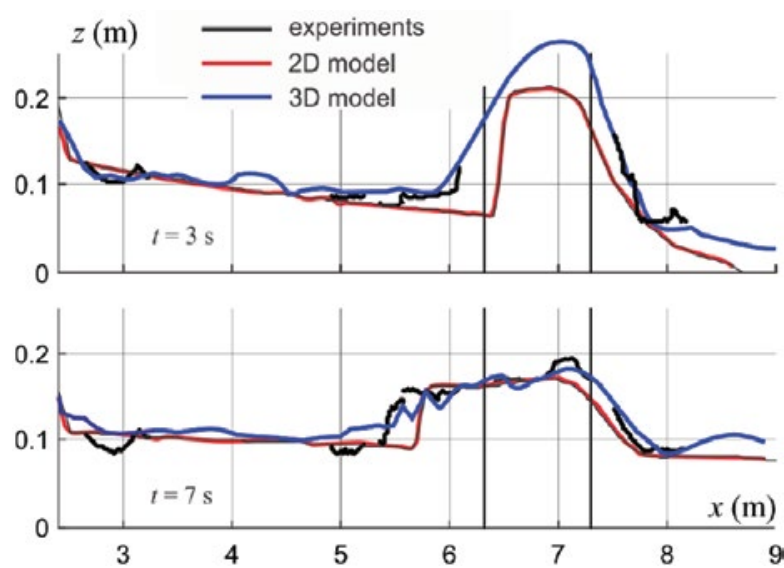

Figure 14 : Developed water profile for $90^{\circ}$ bend channel: experimental observation, $2 D$ and $3 D$ computations.

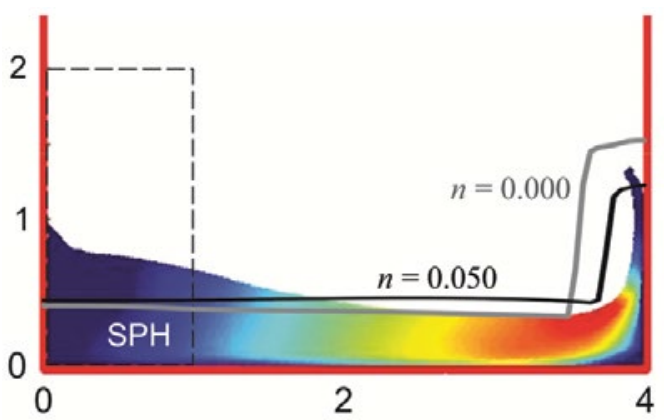

Figure 15: SPH approach (surface) compared to $1 D$ finite-volume computation (lines) $-\boldsymbol{t}=0.88 \mathrm{~s}$.

delivers nice picture of the splashing zone but it fails in representing the emptying of the upstream reservoir: indeed, a portion of water appears as "stuck" to the wall, which is probably again an effect of the excessive artificial viscosity.

\section{II.4. Hybrid approaches and simplified models}

It is clear that each approach presents advantages and drawbacks. Two limiting factors are generally considered: the

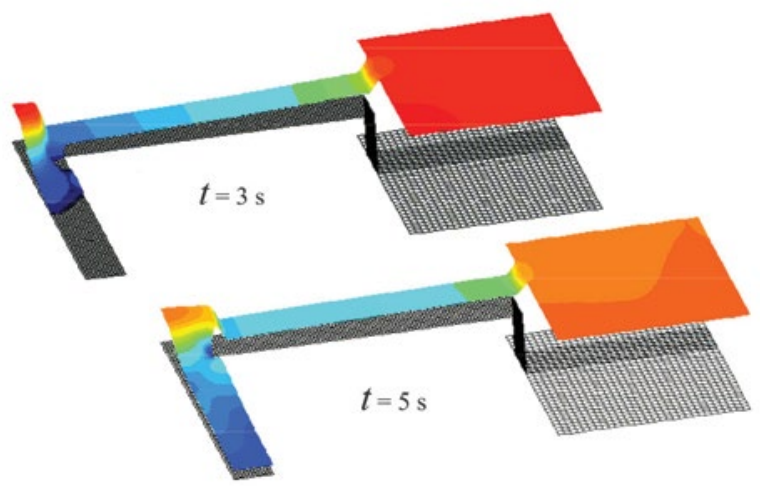

Figure 13 : $2 D$ numerical simulation of the $90^{\circ}$ bend. 
computational time, which is less and less a problem due to the increasing power of computers, and the requirements in terms of data. This latter limitation is an invitation to simplify the non-essential parts of the problem and to use hybrid approaches for refining the results in the area of interest.

An example of a simplified model used for the above-described $90^{\circ}$ bend is shown in Figs. 16 and 17 . From digital imaging measurements, the velocity field in the bend was obtained, which allowed the reconstruction of the stream lines, showing two recirculation zones (Fig. 16). So, a fictitious straight geometry was tested (Fig. 17) featuring the same hydraulic behaviour as the bend of figure 10, i.e. a locally constricted cross-section. Such an analogous constriction allows the use of a 1D model with results (not shown here) rather close to 2D [Soares-Frazão, 2002].

Another way to obtain the same result is the use of hybrid $1 \mathrm{D} / 2 \mathrm{D}$ approaches. The same $90^{\circ}$-bend channel case concentrates most of the 2D features in the bend itself, while the upstream and downstream reaches present rather 1D behaviour. Such a hybrid approach is illustrated in Fig. 18, with results in Fig. 19. These results agree reasonably well with the measurements, except regarding the front celerity.

\section{WHAT HAPPENS OVER MOBILE BED?}

Most of fast-transient flow studies only focus on the hydrodynamic behaviour of the wave. However the amount

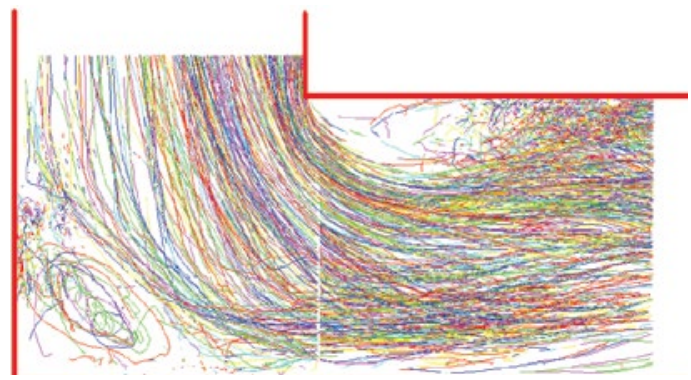

Figure $16: 90^{\circ}$ bend surface trajectories.

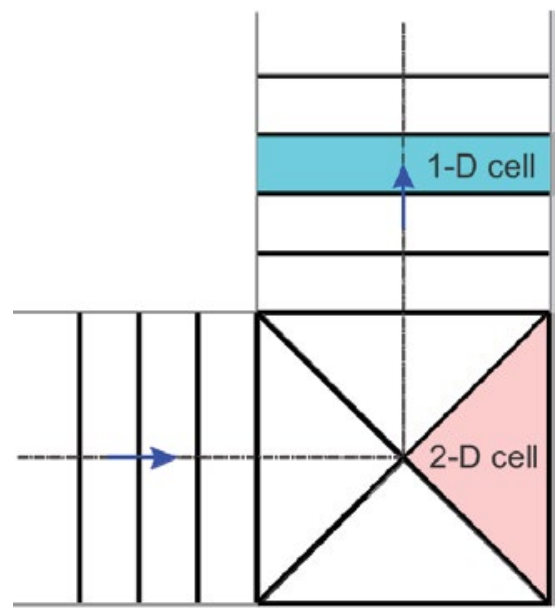

Figure 18 : $90^{\circ}$ bend: hybrid $1 \mathrm{D} / 2 \mathrm{D}$ model. of sediments mobilised by a sudden release of water may be of the same order of magnitude as the volume of released water [Capart, 2000]. Modelling sediment movement induced by severe transients remains a challenge, as most common approaches neglect inertia effects in sudden mobilisation of sediments. Four types of model are generally used: (1) sediment movement instantaneously adapting to hydrodynamic changes, (2) spatial or temporal lag laws to give space or time to the sediments to progressively reach the transport capacity, (3) two-phase model allowing the sediments to move independently of the water but inside the water, and (4) two-layer model, able to account for the inertia of the bed-load sediment layer. The first model has proved to be inaccurate in fast transient flows, while the lag laws are difficult to calibrate. So the two last approaches seem the most promising. Here we propose to focus on the two-layer model that is developed from years in the authors' research team and thus more familiar to them.

\section{III.1. Two-layer (2L) approach: basic model}

This approach was initially proposed by Capart [2000]. Extensions of the model have been proposed by Capart and Young [2002], Spinewine [2005] and Savary [2007]. Assuming a shallow-water hydrostatic pressure distribution, the flow is divided in two layers. The upper layer is made of flowing water only. The lower flow layer materialises the sediment transport layer as a sheet flow. The main variables

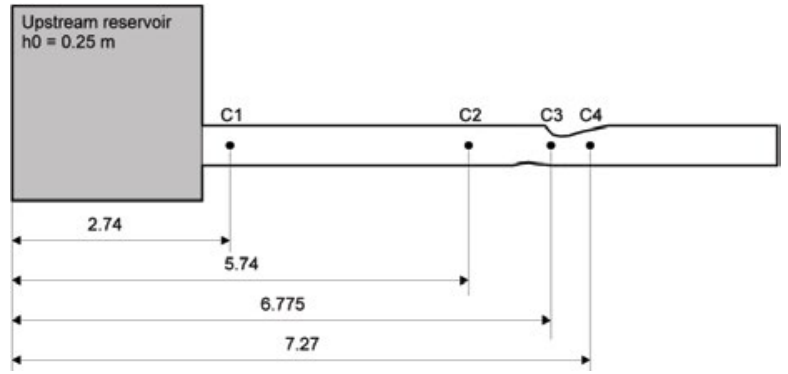

Figure 17 : Analogous constriction for the $90^{\circ}$ bend.
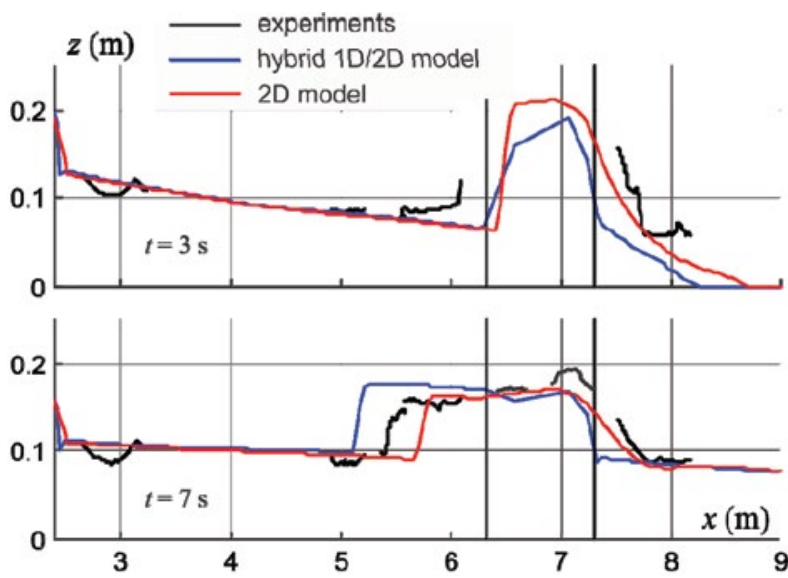

Figure 19 : $90^{\circ}$ bend: comparison between experiments and models. 
are the thickness of the water layer $h_{w}$ and of the transport layer $h_{s}$, the velocity in each layer $u_{w}$ and $u_{s}$, respectively, and the level of the interface between the transport layer and the bed $z_{b}$. The sediment bed has a volumetric sediment concentration $C_{b}$, while the transport layer is made of a water-sediment mixture with a sediment concentration $C_{s}$. Both $C_{b}$ and $C_{s}$ are assumed constant in time and in space (Fig. 20(a)). These assumptions, while not completely in agreement with observations, make the model simpler without affecting the essential features of the involved mechanisms.

Defining the downwards movement of the interfaces $\Gamma_{b}$ and $\Gamma_{s}$ as $e_{b}$ and $e_{s}$, respectively

$$
\frac{\partial z_{b}}{\partial t}=-e_{b} \text { and } \frac{\partial z_{s}}{\partial t}=-e_{s}
$$

it is possible to deduce a relation between both [Savary, 2007] considering the vertical mass balance. For instance, in case of erosion (Fig. 20(b): $e_{b}>0$ ), the interface $\mathrm{G}_{b}$ has moved down, in such a way that the volume $\mathrm{DV}_{2}$ that was in the bottom layer is now in the transport layer, so decreasing in density with more void to be filled by water. This water is taken from the upper layer, the volume $\Delta \mathrm{V}_{1}$ moves from the clear water to the transport layer, with an increase of density compensating $\Delta \mathrm{V}_{2}$. Consequently, the interface $\mathrm{G}_{s}$ moves up with a rate $e_{s}$ depending on $e_{b}$. So, $e_{s}$ and $e_{b}$ are not independent and only one has to be considered.

Accounting for the fact that horizontal boundaries move with deposition or erosion, conservation of mass in control volumes of Figs.20(c-d) read, [Zech et al., 2007]:

$$
\frac{\partial h_{w}}{\partial t}+\frac{\partial h_{w} u_{w}}{\partial x}=e_{s} \text { and } \frac{\partial h_{s}}{\partial t}+\frac{\partial h_{s} u_{s}}{\partial x}=e_{b}-e_{s}
$$

while conservation of momentum applied to the same control volumes reads:

$$
\frac{\partial h_{w} u_{w}}{\partial t}+\frac{\partial}{\partial x}\left(h_{w} u_{w}^{2}+\frac{1}{2} g h_{w}^{2}\right)+g h_{w} \frac{\partial\left(z_{b}+h_{s}\right)}{\partial x}=u_{w} e_{s}-\frac{\tau_{w s}}{\rho_{w}}
$$

$$
\begin{aligned}
& \frac{\partial h_{s} u_{s}}{\partial t}+\frac{\partial}{\partial x}\left(h_{s} u_{s}^{2}+\frac{1}{2} g h_{s}^{2}\right)+g h_{s} \frac{\partial}{\partial x}\left(z_{b}+\frac{\rho_{w}}{\rho_{s}{ }^{\prime}} h_{w}\right) \\
& =-\frac{\rho_{w}}{\rho_{s}{ }^{\prime}} u_{w} e_{s}+\frac{\tau_{w s}}{\rho_{s}{ }^{\prime}}-\frac{\tau_{b}}{\rho_{s}{ }^{\prime}}
\end{aligned}
$$

Equations (16-19) together with equation (14) form a system of five equations with five unknowns: $h_{w}, h_{s}, z_{b},\left(h_{w} u_{w}\right)$ and $\left(h_{s} u_{s}\right)$. Expressing the momentum conservation in the elementary control volume of Fig. 20(e), it is possible to link $e_{b}$ to the shear stresses:

$$
e_{b}=\frac{\tau_{s}-\tau_{b}}{\rho_{b} u_{s}}
$$

which implies a discontinuity in velocity and shear stress distributions at the lower interface of transport layer. The other parameters $\left(\tau_{w s}, \tau_{s}, \tau_{b}\right)$ require three closure equations. The shear stresses $\tau_{w s}$ and $\tau_{s}$ are evaluated from the turbulent friction:

$$
\tau_{w s}=\rho_{w} C_{f, w}\left|u_{w}-u_{s}\right|\left(u_{w}-u_{s}\right) \text { and } \tau_{s}=\rho_{s}{ }^{\prime} C_{f, s}\left|u_{s}\right| u_{s}
$$

while $\tau_{b}$ depends on a critical shear stress $\tau_{c}$, and the internal friction angle $\phi$ :

$$
\tau_{b}=\tau_{c}+\left(\rho_{s}^{\prime}-\rho_{w}\right) g h_{s} \tan \varphi
$$

where $\rho_{s}^{\prime}=\left(1-C_{s}\right) \rho_{w}+C_{s} \rho_{s}$, is the volumetric density of the transport layer, $\rho_{w}$ and $\rho_{s}$ being the water and grain density, respectively. Two coefficients remain to be calibrated from experiments: $C_{f, w}$, and $C_{f, s}$.

Some of the above assumptions are not completely in agreement with experimental observations: neither the velocity $u$, nor the concentration $C$ are really constant in the transport layer, they rather obey to a linear distribution. Also the discontinuity in velocity at the interface, which allows writing (20) is not observed. However, despite these limitations, the simulation of the dam-break flow case illustrated in Fig. 21 is rather accurate (Fig. 22).

The two-layer model as presented above is easily extended to two-dimensional approach, just using components of velocities and shear stresses. Complete theory and some applications may be found in Swartenbroekx et al. [2013]. Among other, cases of dam-break flow over mobile bed with a sudden enlargement of the valley (e.g. Goutière et al. 2011 or Soares-Frazão et al. 2012) take advantage of the possibility to compute distinct sediment and water velocity directions.

\section{III.2. Two-layer approach: perspectives}

Using new developments of imaging techniques, Spinewine and Capart [2013] carried out measurements
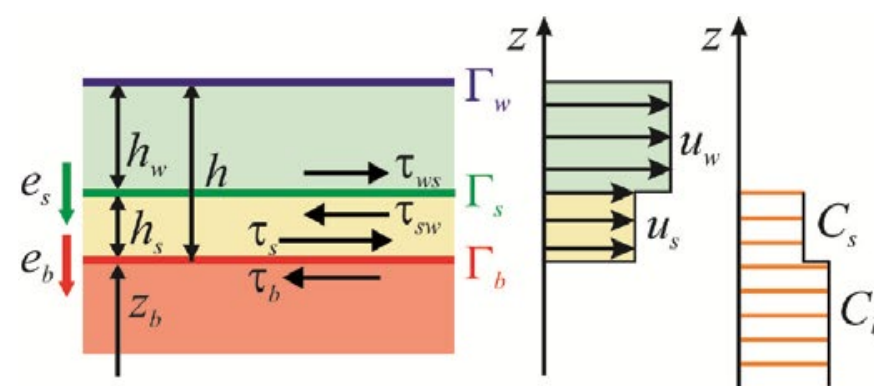

(a)

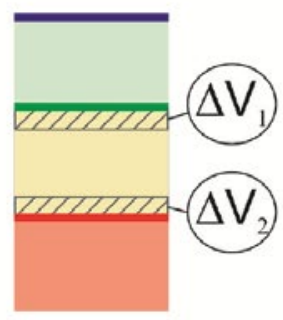

(b)

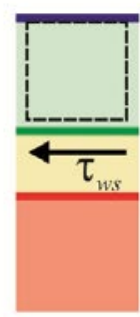

(c)

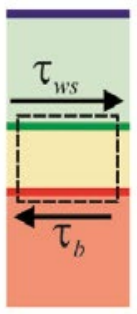

(d)

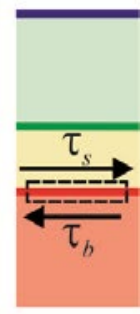

(e)

Figure 20 : Two-layer approach. 


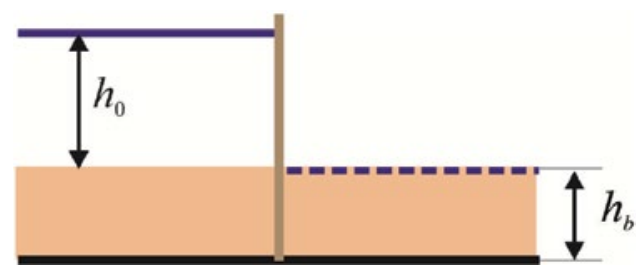

Sand bed

$h_{0}=0.350 \mathrm{~m}$

$C_{f, w}=0.005$

$h_{b}=0.125 \mathrm{~m}$

$C_{f, s}=0.040$

Figure 21 : Dam-break experimental set-up.

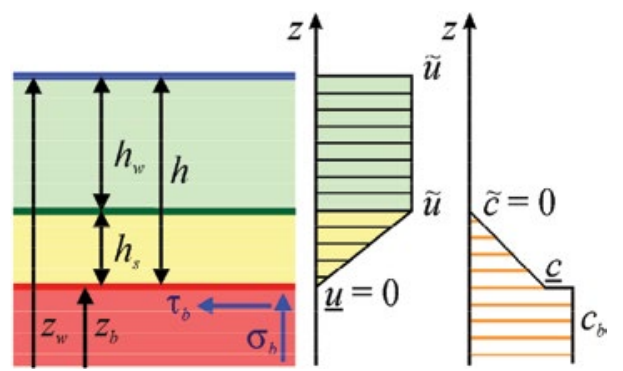

Figure 23 : Improved $2 L$ approach with linear distribution.

not only of the velocity profile in the transport layer but also of the concentration distribution, confirming that both are linear. They also developed a two-layer model in line with these observations (Fig. 23). Now $\underline{c}$, the concentration at the bottom interface of the transport layer, enters the set of variables in the equations, avoiding to arbitrarily impose this value. Fig. 24 shows the case of a dam-break with $h_{0}=0.35 \mathrm{~m}$, over PVC pellets $\left(D_{\text {equ }}=3.92 \mathrm{~mm}\right.$, $\left.\rho_{s}=1580 \mathrm{~kg} / \mathrm{m}^{3}\right)$, comparing the measured and computed velocity and concentration profiles. The new scheme proves to be really promising. Two obstacles however remain in that kind of model: on the one hand, in a 2D transposition of the model, the sediment transport velocity is now forced to have the same direction as the water velocity, which is not realistic; on the other hand, closure equations for the bottom stresses $\sigma_{b}$ and $\tau_{b}$ are not evident and probably require more experimental investigations to better understand what happens along the layer interfaces.

\section{CONCLUSIONS}

At the end of this overview, it seems clear that each approach - 1D, 2D, 3D - presents advantages and drawbacks. Hybrid approaches are thus promising as they ensure accuracy where it is required, without demanding too much in terms of data.

Regarding the modelling of intense erosion and deposition generally induced by severe transients, several approaches seem promising, none of them completely founded physically, so requiring further research.

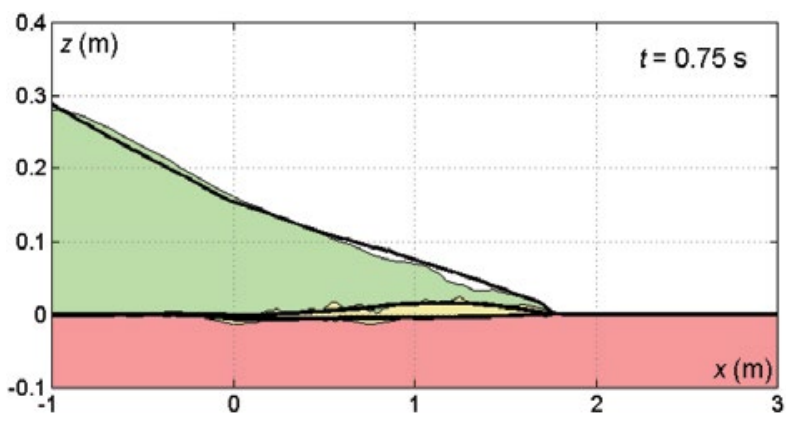

Figure 22 : Comparison experiments and two-layer model.
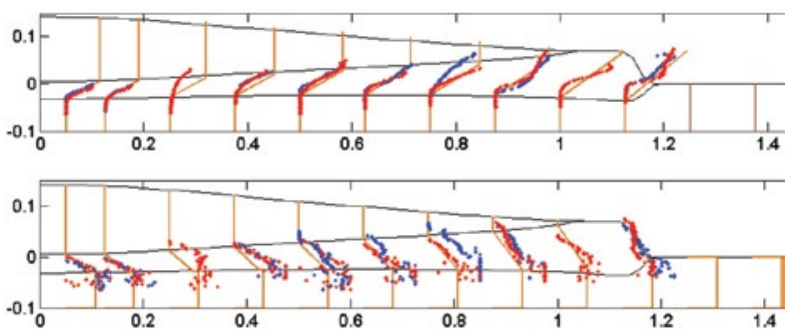

Figure 24 : Measured (points) and computed (lines) velocity (above) and concentration (below) profiles.

\section{REFERENCES}

Aleixo R., SoAres-FraZão S. And ZeCh Y. (2011) - Velocity-field measurements in a dam-break flow using a PTV Voronoï imaging technique. Experiments in Fluids 50(6) 1633-1649

Aleixo R., Soares-Frazão S., Altinakar M. And Zech Y. (2013) - The Gate Removal Effect in the Initial Instants of the Dam-break Flow. Proceedings $35^{\text {th }}$ IAHR Congress, Chengdu, China, USB-key. 10 pages

Bermùdez A. ANd VÁzquez M.E. (1994) - Upwind methods for hyperbolic conservation laws with source terms. Computers and Fluids. 23(8) 1049-1071

Biscarini C. Di Francesco S. And Manciola P. (2010) - CFD modelling approach for dam break flow studies. Hydrol. Earth Syst. Sci. 14 705-718

Braschi G. And Gallati M. (1992) - A conservative flux prediction algorithm for the explicit computation of transcritical flow in natural streams, Proc. Hydr. Eng. Software IV, CMP, Southampton. 381-394

CAPART H. (2000) - Dam-break induced geomorphic flows and the transition from solid- to fluid-like behaviour across evolving interfaces. PhD thesis, UCL, Belgium

CAPART H., And Young D.L. (2002) - Two-layer shallow water computations of torrential geomorphic flows. Proc. River Flow 2002, Louvain-la-Neuve, Belgium. Balkema, Rotterdam, Netherlands. 1003-1012

FERZIGER J.H. AND PERIĆ M. (2002) — Computational methods for fluid dynamics Springer, Heidelberg

Gomez-Gesteira M., Rogers B.D., Dalrymple R.A. And CRESPo A.J.C. (2010) - State-of-the-art of classical SPH for free-surface flows. Journal of Hydraulic Research. 48(S1) 6-27 
Goutiere L., Soares-Frazão S., Zech Y. (2011) - Dam-break flow on mobile bed in abruptly widening channel: experimental data. Journal of Hydraulic Research. 49(3) 367-371

Guinot V. (2008) - Wave propagation in fluids. Models and numerical techniques. ISTE-Wiley

Harten A., Lax P.D. And Van Leer B. (1983) - On upstream differencing and Godunov-type schemes for hyperbolic conservation laws. J. Comput. Phys. 50 235-269

PATANKAR S.V. (1980) - Numerical heat transfer and fluid flow. Taylor \& Francis

Petaccia G., Natale L., Savi F., Velickovic M., Zech Y. And. SOARES-FraZÃo S. (2013) - Flood wave propagation in steep mountain rivers. Journal of Hydroinformatics. 15(1) 120-137

RoE P. L. (1981) — Approximate Riemann solvers, parameter vectors and difference scheme. J. Comp. Phys. 43 357-372

SAVARY C. (2007) - Transcritical transient flow over mobile beds. Boundary conditions treatment in a two-layer shal-low-water model. PhD thesis, UCL, Belgium

SoARES-FrazÃo S. (2002) - Dam-break induced flows in complex topographies. Theoretical, numerical and experimental approaches. PhD thesis, UCL, Belgium

SoARES-FrazÃo S. AND ZECH Y. (2002) - Dam-break in channels with $90^{\circ}$ bend. Journal of Hydraulic Engineering. 128(11) 956-968

Soares-Frazão S., Lories D., Taminiau S. And Zech Y. (2003) - Dam-break flow in a channel with a sudden enlargement. Proceedings $30^{\text {th }}$ IAHR Congress, Thessaloniki, Greece. C-II 221-228
SoARES-FrazÃo S. AND GuINOT V. (2007) - An eigenvector-based linear reconstruction scheme for the shallow-water equations on two-dimensional unstructured meshes. Int. J. Numer. Meth. Fluids. 53(1) 23-55

Soares-Frazão S., Canelas R., Cao Z., Cea L., Chaudhry H., Die Moran A., El Kadi K., Ferreira R., Cadórniga I.F., Gonzalez-Ramirez N., Greco M., Huang W., Imran J., Le Coz J., Marsooli R., Paquier A., Pender G., Pontillo M., Puertas J., Spinewine B., Swartenbroekx C., Tsubaki R., Villaret C., Wu W., Yue Z. And Zech Y. (2012) Dam-break flows over mobile beds: Experiments and benchmark tests for numerical models. Journal of Hydraulic Research. 50(4) 364-375

SPINEwINE B. (2005) - Two-layer flow behaviour and the effects of granular dilatancy in dam-break induced sheet-flow. $P h D$ thesis, UCL, Belgium

SPINEwine B. AND CAPART H. (2013) - Intense bed-load due to a sudden dam-break. Journal of Fluid Mechanics. 731 579-614

Swartenbroekx C., Zech Y. And Soares-FrazÃo S. (2013) Two-dimensional two-layer shallow water model for dam break flows with significant bed load transport Int. J. Numer. Meth. Fluids. 73(5) 477-508

ToRo E.F. (1997) - Riemann Solvers and Numerical Methods for Fluid Dynamics. A practical introduction. Springer-Verlag, Berlin, Germany

Zech Y., Soares-Frazão S., Spinewine B., Savary C. And Goutière L. (2009) - Inertia effects in bed-load transport models. Canadian Journal of Civil Engineering. 36 (10) 1587-1597 